\title{
Bragg spectroscopy of a Bose-Einstein condensate
}

\author{
J. Stenger, S. Inouye, A.P. Chikkatur, D.M. Stamper-Kurn, D.E. Pritchard, and W. Ketterle \\ Department of Physics and Research Laboratory of Electronics, \\ Massachusetts Institute of Technology, Cambridge, MA 02139
}

February 1, 2008

\begin{abstract}
Properties of a Bose-Einstein condensate were studied by stimulated, two-photon Bragg scattering. The high momentum and energy resolution of this method allowed a spectroscopic measurement of the mean-field energy and of the intrinsic momentum uncertainty of the condensate. The coherence length of the condensate was shown to be equal to its size. Bragg spectroscopy can be used to determine the dynamic structure factor over a wide range of energy and momentum transfers.
\end{abstract}

The first evidence for Bose-Einstein condensation in dilute gases was obtained by a sudden narrowing of the velocity distribution as observed for ballistically expanding clouds of atoms [1]. Indeed, most textbooks describe Bose-Einstein condensation as condensation in momentum space [2]. However, the dominant contribution to the observed momentum distribution of the expanding condensate was the released interaction energy (meanfield energy) resulting in momentum distributions much broader than the zero-point motion of the ground state of the harmonic trapping potential. Since the size of a trapped condensate with repulsive interactions is larger than the trap ground state, the momentum distribution should be considerably narrower than in the trap ground state. In this paper, we show the momentum distribution of a trapped condensate to be Heisenberg uncertainty limited by its finite size. This is equivalent to showing that the coherence length of the condensate is equal to its physical size.

Sub-recoil momentum resolution has been previously achieved by resolving the Doppler width of a Raman transition between different hyperfine states [3] or of a twophoton transition to a metastable excited state 胉. Here we use Bragg scattering where two momentum states of the same ground state are connected by a stimulated two-photon process [5]. This process can be used to probe density fluctuations of the system and thus to measure directly the dynamic structure factor $\mathbf{S}(\mathbf{q}, \nu)$, which is the Fourier transform of the density-density correlation function and is central to the theoretical description of many-body systems [6]. In contrast to measuring $\mathrm{S}(\mathbf{q}, \nu)$ with inelastic neutron scattering like in superfluid helium [7], or using inelastic light scattering [8].9], Bragg scattering as used here is a stimulated process which greatly enhances resolution and sensitivity.

Bragg scattering of atoms from a light grating was first demonstrated in 1988 [10] and has been used to manipulate atomic samples in atom interferometers [1], in de Broglie wave frequency shifters [12], and also to couple out or manipulate a Bose-Einstein condensate [5]. Small angle Bragg scattering, called recoil-induced res- onances, has been used for thermometry of laser-cooled atoms [13]. In this work we establish Bragg scattering as a spectroscopic technique to probe properties of the condensate. We refer to it as Bragg spectroscopy in analogy to Raman spectroscopy which involves different internal states.

The absorption of $N$ photons from one laser beam and stimulated emission into a second laser beam constitutes an $N$-th order Bragg scattering process. The momentum transfer $q$ and energy transfer $h \nu$ are given by $q=2 N \hbar k \sin (\vartheta / 2)$ and $\nu=N \Delta \nu$, where $\vartheta$ is the angle between the two laser beams with wave vector $k$ and frequency difference $\Delta \nu$.

For non-interacting atoms with initial momentum $\hbar k_{i}$, the resonance is given by the Bragg condition $h \nu=$ $q^{2} / 2 m+\hbar k_{i} q / m$, which simply reflects energy and momentum conservation for a free particle. The second term is the Doppler shift of the resonance and allows the use of Bragg resonances in a velocity-selective way [5, 13 .

For a weakly interacting homogeneous condensate at density $n$, the dispersion relation has the Bogoliubov form [2]

$$
\nu=\sqrt{\nu_{0}^{2}+2 \nu_{0} n U / h}
$$

where $n U=n 4 \pi \hbar^{2} a / m$ is the chemical potential, with $a$ and $m$ denoting the scattering length and the mass, respectively, and $h \nu_{0}=q^{2} / 2 m$. At low energies the excitation spectrum is phonon-like, obeying $h \nu=c q$, where $c$ is the speed of sound [2]. For energies $h \nu \gg n U$ the spectrum is particle-like

$$
\nu \approx \nu_{0}+n U / h .
$$

The mean-field shift $n U$ reflects the exchange term in the interatomic interactions: a particle with momentum $q$ experiences twice the mean field energy as a particle in the condensate [2]. We use this property to determine the condensate mean-field energy spectroscopically. This is related to, but different from the mean-field shift due to interactions with an electronically excited state which was used to identify BEC in atomic hydrogen 朋. 
Eq. (11) is the excitation spectrum of a homogeneous condensate with initial momentum $\hbar k_{i}=0$. The inhomogeneous trapping potential adds two features which broaden the resonance: a distribution of initial momenta due to the finite size of the cloud, and a distribution of mean-field shifts due the density variation over space.

The momentum distribution along the $\mathrm{x}$-axis is given by the Fourier transform of the wavefunction: $f\left(p_{x}\right)=$ $\left[\int d x d y d z e^{-i p_{x} x / \hbar} \Psi(x, y, z)\right]^{2}$. In the Thomas-Fermi approximation the wave function $\Psi(x, y, z)$ in an harmonic trapping potential is $[\Psi(x, y, z)]^{2}=n_{0}(1-$ $\left.\left(x / x_{0}\right)^{2}-\left(y / y_{0}\right)^{2}-\left(z / z_{0}\right)^{2}\right)$, where $n_{0}$ denotes the peak density. The size of the wavefunction is determined by $n_{0}$ and the trapping frequencies $\nu_{i}$ through the coefficients $x_{0}, y_{0}, z_{0}: x_{0}=\sqrt{2 n_{0} U / m\left(2 \pi \nu_{x}\right)^{2}}$ (and similar for $\left.y_{0}, z_{0}\right)$. The line shape $I_{p}\left(p_{x}\right)$ is proportional to the square of the Fourier coefficients [14]

$$
I_{p}\left(p_{x}\right) \sim\left[J_{2}\left(p_{x} x_{0} / \hbar\right) /\left(p_{x} x_{0} / \hbar\right)^{2}\right]^{2}
$$

where $J_{2}$ denotes the Bessel function of order 2. This curve is very similar to a Gaussian and has an rms-width of $\Delta p_{x}=\sqrt{21 / 8} \hbar / x_{0}$. Thus, the corresponding Doppler broadening $\Delta \nu_{p}=\sqrt{21 / 8} q / 2 \pi m x_{0}$ of the Bragg resonance is inversely proportional to the condensate size $x_{0}$ and does not depend explicitly on the number of atoms.

The same parabolic wavefunction gives the (normalized) density distribution $\left(15 n / 4 n_{0}\right) \sqrt{1-n / n_{0}}$. The simplest model for the spectroscopic lineshape $I_{n}(\nu)$ due to the inhomogenous density assumes that a volume element with local density $n$ leads to a lineshift $n U$ (eq. (2)):

$$
I_{n}(\nu)=\frac{15 h\left(\nu-\nu_{0}\right)}{4 n_{0} U} \sqrt{1-\frac{h\left(\nu-\nu_{0}\right)}{n_{0} U}}
$$

which has a total width of $n_{0} U / h$, a maximum at $2 n_{0} U / 3 h$, an average value (first moment) of $4 n_{0} U / 7 h$, and an rms-width of $\Delta \nu_{n}=\sqrt{8 / 147} n_{0} U / h$. In contrast to the Doppler broadening due to the finite size, the mean-field broadening only depends on the density but not explicitly on the size.

In our experiments the combined broadening mechanisms represented by eqs. (3) and (4) have to be considered. While the exact calculation of the lineshape requires detailed knowledge of the excitation wavefunctions, the total line shift (first moment) and rms-width can be calculated using sum rules and Fermi's Golden Rule. Thus, it can be rigorously shown that the total line shift remains $4 n_{0} U / 7 h$, while the rms-widths $\Delta \nu=\sqrt{\Delta \nu_{p}^{2}+\Delta \nu_{n}^{2}}$ is the quadrature sum of the Doppler and mean-field widths 15 .

We produced magnetically trapped, cigar-shaped Bose-Einstein condensates as in previous work [16]. In order to study the resonance as a function of density and size, we prepared condensates using two different trapping frequencies and varied the number of atoms by removing a variable fraction using the rf-output coupler [17]. The density of the condensate was determined from the expansion of the cloud in time-of-flight and the size from the atom number and the trapping frequencies [16]. Bragg scattering was performed by using two counterpropagating beams aligned perpendicularly to the weak axis of the trap. Spectra were taken by pulsing on the light shortly before switching off the trap and determining the number of scattered atoms as a function of the frequency difference between the two Bragg beams. Since the kinetic energy of the scattered atoms was much larger than the mean-field energy, they were well separated from the unscattered cloud after a typical ballistic expansion time of $20 \mathrm{msec}$. Center frequencies and widths were determined from Gaussian fits to the spectra.

Duration, intensity and detuning of the Bragg pulses had to be chosen carefully. The instrumental resolution is limited by the pulse duration $\delta t_{\text {pulse }}$ due to its Fourier spectrum, in our case requiring $\delta t_{\text {pulse }}>250 \mu \mathrm{s}$ for sub- $\mathrm{kHz}$ resolution. The maximum pulse duration was limited to less than one quarter of the trap period, $\delta t_{\text {pulse }}<500 \mu \mathrm{s}$, by which time the initially scattered atoms would come to rest and thus would be indistinguishable from the unscattered atoms in time-of-flight. The light intensity was adjusted to set the peak efficiency to about $20 \%$. Sufficient detuning was necessary to avoid heating of the sample. The ratio of the two-photon rate $\omega_{R}^{2} / 4 \Delta$ to the spontaneous scattering rate $\omega_{R}^{2} \Gamma / 2 \Delta^{2}$ is $\Gamma / 2 \Delta$, where $\omega_{R}$ denotes the single beam Rabi frequency, $\Delta$ the detuning and $\Gamma$ the natural linewidth. Spontaneous scattering was negligible for the chosen detuning of $1.77 \mathrm{GHz}$ below the $3 \mathrm{~S}_{1 / 2} \mathrm{~F}=1 \rightarrow 3 \mathrm{P}_{3 / 2} \mathrm{~F}=2$ transition.

The relative detuning of the two Bragg beams was realized in two ways. In one scheme, a beam was split and sent through two independent acousto-optical modulators driven with the appropriate difference frequency, and then overlapped in a counterpropagating configuration. Alternatively, a single beam was modulated with two frequencies separated by the relative detuning and backreflected. Both methods are insensitive to frequency drifts of the laser since the Bragg process only depends on the relative frequency of the two beams, which is controlled by $\mathrm{rf}-$-synthesizers. The second method simultaneously scattered atoms in the $+x$ and $-x$ directions and was thus helpful to identify motion of the cloud. We estimate that residual vibrational noise broadened the line by less than $1 \mathrm{kHz}$. This resolution corresponds to a velocity resolution of $0.3 \mathrm{~mm} / \mathrm{s}$ or $1 \%$ of the single-photon recoil. At a radial trapping frequency of $200 \mathrm{~Hz}$, we had to avoid any motion of the cloud with an amplitude larger than $0.2 \mu \mathrm{m}$.

Fig. 1 shows typical spectra, taken both for a trapped condensate and after $3 \mathrm{~ms}$ time of flight when the mean- 
field energy was fully converted into kinetic energy. The rms-width of the resonance for the ballistically expanding cloud is $20 \mathrm{kHz}$, which is much narrower than the $65 \mathrm{kHz}$ wide distribution of a thermal cloud at $1 \mu \mathrm{K}$, a typical value for the BEC transition temperature under our conditions.

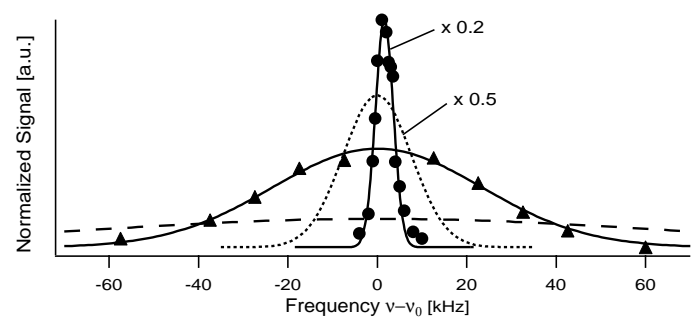

FIG. 1. Bragg resonances for a trapped condensate (circles) and after $3 \mathrm{~ms}$ time of flight (triangles). For comparison, the momentum distributions of the ground state of the trapping potential (dots) and of a $1 \mu \mathrm{K}$ cold, thermal cloud (dashes) are indicated.

We could not measure the thermal distribution with the same pulse duration as for the condensate since the fraction of scattered atoms was too small due to the broad resonance. The spectra for the thermal cloud and the expanding condensate correspond to the spatial distributions observed by absorption imaging after sufficiently long time of flight. With this technique, the BEC transition is indicated by a sudden narrowing of the time-offlight distribution by a factor of three. Using Bragg spectroscopy, the signature of BEC is much more dramatic - the condensate resonance is more than thirty times narrower than of the thermal cloud, and indeed narrower than the ground state of the trap. This demonstrates that Bragg spectroscopy is a superior way to probe the BEC transition.

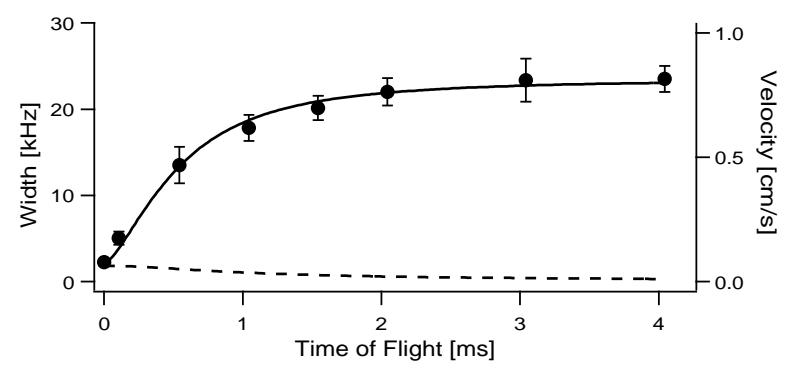

FIG. 2. Mean-field acceleration of a condensate released from the trap. Shown is the increase of the width of the Bragg resonance during the expansion. The solid line is the theoretical prediction [18] using the trap frequency $\nu_{r}=195 \mathrm{~Hz}$. The dashed line represents the contributions of mean-field energy and finite size to the total width.

Fig. 2 shows the conversion of mean-field energy into kinetic energy after the atoms are released from the trap, indicated by a broadening of the Bragg resonance from about $2 \mathrm{kHz}$ to $20 \mathrm{kHz}$. After $3 \mathrm{~ms}$, the cloud has reached its asymptotic velocity $v_{\infty}$. Fig. 2 agrees with the velocity evolution $v_{r}=v_{\infty} 2 \pi \nu_{r} t / \sqrt{1+\left(2 \pi \nu_{r} t\right)^{2}}$ as expected from the scaling laws given in ref. [18] which are derived for cigar shaped condensates with large aspect ratios in the Thomas-Fermi approximation. In order to compare with the data, the Doppler width due to the velocity evolution, the mean-field width and the finite-size width were added in quadrature, assuming Gaussian shapes. The finite-size width was calculated from the predicted evolution of the size $x_{r}=x_{0} \sqrt{1+\left(2 \pi \nu_{r} t\right)^{2}}$ [18.

The narrow resonance of the trapped condensate (Fig. 1) was studied as a function of the condensate density and size. Fig. 3 (a) demonstrates the linear dependence of the frequency shift on the density. The slope of the linear fit corresponds to $(0.54 \pm 0.07) n_{0} U / h$, in excellent agreement with the prediction of $4 n_{0} U / 7 h$. In Fig. 3 (b), the expected widths due to the mean-field energy and finite size are shown for the two different trapping frequencies studied. The data agree well with the solid lines, which represent the quadrature sum of the two contributions. To demonstrate the finite-size effect the same data are shown in Fig. 3 (c) after subtracting the meanfield broadening and the finite pulse-length broadening $(0.5 \mathrm{kHz})$. The linewidths are consistent with the expected $1 / x_{0}$ dependence. Even without these corrections the measured linewidths are within $20 \%$ of the value expected due to the Heisenberg-uncertainty limited momentum distribution (Fig. 3 (b)).

The momentum spread of the condensate is limited by its coherence length $x_{c}$ which, in the case of long-range order, should be equal to the size $x_{0}$ of the condensate. Our results show that $x_{c} \approx x_{0}$ in the radial direction of the trap. This quantitatively confirms the earlier qualitative conclusion reached by interfering two condensates [19. In particular, our measurements indicate that the condensate does not have phase fluctuations, i.e. that it does not consist of smaller quasi-condensates with random relative phases. It would be interesting to study this aspect during the formation of the condensate, e.g. after suddenly quenching the system below the BEC transition temperature 20], and observe the disappearance of phase fluctuations.

In this work we have determined the normal mode of the condensate at a momentum transfer of two photon recoils, corresponding to a energy transfer $\nu_{0}=100 \mathrm{kHz}$. Different momentum transfers are possible by changing the angle between the Bragg beams and/or the order $N$, thus enabling measurements of the dynamical structure function $S(\mathbf{q}, \nu)$ over a wide range of parameters. At low momentum transfer, the lineshape is dominated by the mean-field energy and by phonon-like collective excitations, whereas at high momentum transfers, the linewidth mainly reflects the momentum distribution of individual atoms. This is analogous to neutron scattering in liquid helium, where slow neutrons were used to observe the phonon and roton part of the dispersion 
curve, and fast neutrons were used to determine the zeromomentum peak of the condensate [7]. While we have observed higher-order Bragg scattering up to third order in the trapped condensate using higher laser intensities, its spectroscopic use was precluded by severe Rayleigh scattering, and would require larger detuning from the atomic resonance.
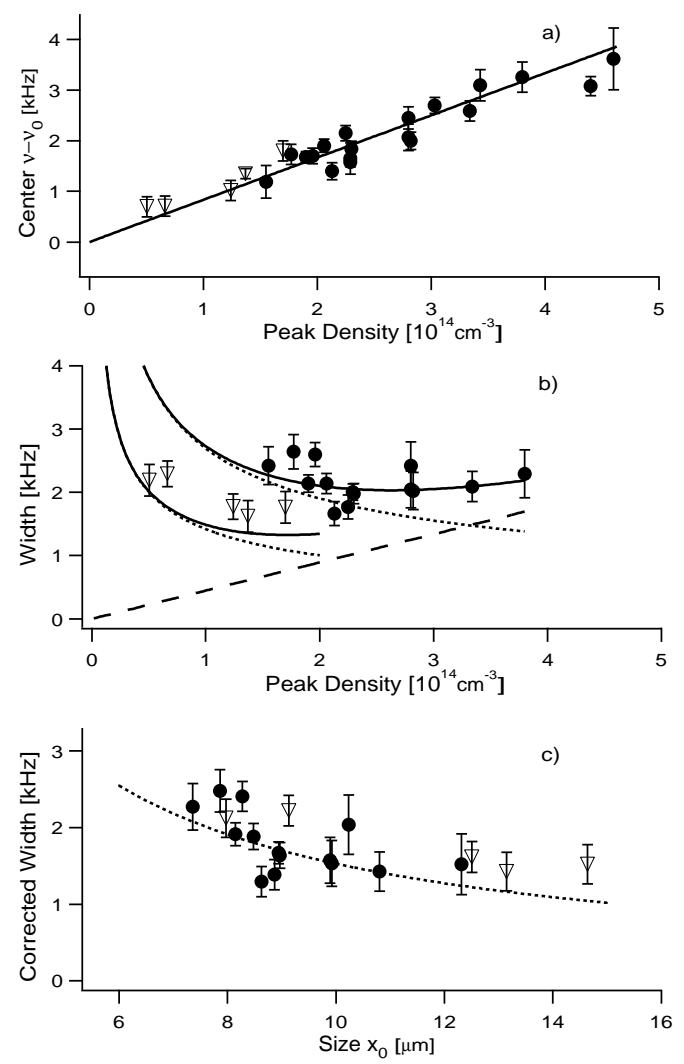

FIG. 3. Bragg spectroscopy of a trapped condensate. Line shifts and widths are shown for various densities and sizes of the condensate using two different radial trapping frequencies, $\nu_{r}=(195 \pm 20) \mathrm{Hz}$ (circles), and $\nu_{r}=(95 \pm 20) \mathrm{Hz}$ (triangles). The lines in b) show the contributions of the mean-field energy (dashed), due to the finite size (dotted, for both trapping frequencies), and their quadrature sum (solid lines). Fig c) displays the width after subtraction of the contributions of the mean-field and the finite pulse duration and compares it with the prediction for the momentum uncertainty due to the finite size. The error bars are $1 \sigma$ errors of the Gaussian fits to the data.

The use of inelastic light scattering to determine the structure factor of a Bose-Einstein condensate was discussed in several theoretical papers [8,9]. It would require the analysis of scattered light with $\mathrm{kHz}$ resolution and suffers from a strong background of coherently scattered light [9]. Bragg spectroscopy has distinct advantages because it is a stimulated, background-free process in which the momentum transfer and energy are pre-determined by the laser beams rather than post-determined from measurements of the momentum and energy of the scat- tered particle.

In conclusion, we have established Bragg spectroscopy as a new tool to measure properties of a condensate with spectroscopic precision. We have demonstrated its capability to perform high-resolution velocimetry by resolving the narrow momentum distribution of a trapped condensate and by observing the acceleration phase in ballistic expansion. Since the momentum transfer can be adjusted over a wide range, Bragg spectroscopy can be used to probe such diverse properties as collective excitations, mean-field energies, coherence properties, vortices or persistent currents.

This work was supported by the Office of Naval Research, NSF, Joint Services Electronics Program (ARO), NASA, and the David and Lucile Packard Foundation. A.P.C would like to acknowledge support from NSF, D.M.S.-K. from the JSEP Graduate Fellowship Program and J.S. from the Alexander von Humboldt-Foundation.

[1] M.H. Anderson et al.; Science269, 198 (1995); K.B. Davis et al.; Phys. Rev. Lett. 75, 3969 (1995).

[2] K. Huang; Statistical Mechanics, 2nd ed. (Wiley, New York, 1987).

[3] M. Kasevich et al.; Phys. Rev. Lett. 66, 2297 (1991).

[4] D.G. Fried et al.; Phys. Rev. Lett. 81, 3811 (1998). T.C. Killian, et al.; ibid. 81, 3807 (1998);

[5] M. Kozuma, et al.; to appear in Phys. Rev. Lett. 82, (1999).

[6] T.J. Greytak; in Quantum Liquids, ed. by J. Ruvalds and T. Regge, North-Holland Pub. (1978).

[7] P. Sokol; in Bose-Einstein condensation, ed. by A. Griffin, D.W. Snoke, and S. Stringari, Cambridge University Press (1995); P. Nozières and D. Pines; The Theory of Quantum Liquids, (Addison-Wesley, Reading, 1994).

[8] J. Javanainen; Phys. Rev. Lett. 75, 1927 (1995); R. Graham and D. Walls; Phys. Rev. Lett. 76, 1774 (1996); A.S. Parkins and D.F. Walls; Phys. Rep. 303, 1 (1998), and references therein.

[9] H.D. Politzer; Phys. Rev. A 55, 1140 (1997).

[10] P.J. Martin, B.G. Oldaker, A.H. Miklich, and D.E. Pritchard; Phys. Rev. Lett. 60515 (1988).

[11] D.M. Giltner, R.W. McGowan, and S.A. Lee; Phys. Rev. Lett. 75, 2638 (1996).

[12] S. Bernet, M.K. Oberthaler, R. Abfalterer, J. Schmiedmayer, and A. Zeilinger ; Phys. Rev. Lett. 77, 5160 (1996).

[13] J.-Y. Courtois, G. Grynberg, B. Lounis, and P. Verkerk; Phys. Rev. Lett. 72, 3017 (1994).

[14] G. Baym and C.J. Pethick; Phys. Rev. Lett. 76, 6 (1996).

[15] D. Stamper-Kurn; to be published.

[16] M.-O. Mewes et al.; Phys. Rev. Lett. 77, 416 (1996).

[17] M.-O. Mewes et al.; Phys. Rev. Lett. 78, 582 (1997).

[18] Y. Castin and R. Dum; Phys. Rev. Lett. 77, 5315 (1996).

[19] M.R. Andrews et al.; Science 275, 637 (1997).

[20] H.-J. Miesner et al.; Science 279, 1005 (1998); D.M. Stamper-Kurn et al.; Phys. Rev. Letters 81, 2194 (1998). 